\title{
Phänomenologische Beschreibung der langen optischen Gitterschwingungen in zweiatomigen polaren Kristallen des trigonalen, tetragonalen und hexagonalen Systems (auf elektrostatischer Grundlage* ${ }^{*}$
}

\author{
Von Ludwig Merten ** \\ Aus dem Institut für theoretische Physik der Universität Münster (Westf.) \\ (Z. Naturforschg. 15 a, 47-58 [1960]; eingegangen am 28. September 1959)
}

\begin{abstract}
Es wird gezeigt, daß sich die polaren optischen Schwingungen für große Wellenlängen (Grenz. schwingungen) in den genannten Kristalltypen ähnlich wie in kubischen Kristallen phänomenologisch behandeln lassen. Die hierzu benötigten Materialkonstanten sind die Dispersionsfrequenzen und Dielektrizitätskonstanten. Die Frequenzen und Polarisationsrichtungen der insgesamt drei Eigen. schwingungen werden berechnet. Dabei ergibt sich, daß nur eine Eigenschwingung rein transversal ist, während die beiden weiteren nur für bestimmte ausgezeichnete Richtungen rein transversal bzw. rein longitudinal sind. Im Gegensatz zur ersten sind die Frequenzen der beiden letztgenannten auch von der Fortpflanzungsrichtung abhängig.

In einem Anhang werden die Voraussetzungen für die Gültigkeit der Theorie genauer formuliert Als Beispiel eines Gitters, für das die Voraussetzungen erfüllt sind, ist das Wurtzitgitter angeführt.
\end{abstract}

In den Jahren 1950/51 wurde von Huang ${ }^{1}$ gezeigt, daß sich die langen optischen Schwingungen für zweiatomige kubische Kristalle rein phänomenologisch behandeln lassen. Zunächst konnte er nämlich nachweisen, daß die Schwingungen zwei Gleichungen der Form

$$
\begin{aligned}
& \ddot{\mathfrak{w}}=b_{11} \mathfrak{w}+b_{12} \mathfrak{E}, \\
& \mathfrak{F}=b_{21} \mathfrak{w}+b_{22} \mathfrak{F}
\end{aligned}
$$

genügen müssen. Dabei stellt $\mathfrak{w}$ die Differenz der (reduzierten) Schwingungsvektoren der beiderlei Atome dar; $\mathfrak{F}$ und $\mathfrak{B}$ sind die mit den Schwingungen verknüpfte makroskopische Feldstärke bzw. $\mathrm{Po}_{0}$ larisation; $b_{11}, b_{12}, b_{21}, b_{22}$ sind skalare Faktoren, zwischen denen noch die Beziehung $b_{21}=b_{12}$ besteht. Diese Faktoren lassen sich, wie Huang zeigte, durch die Dielektrizitätskonstanten $\varepsilon_{0}$ (statisch), $\varepsilon_{\infty}$ (optisch) und die Dispersionsfrequenz $\omega_{0}$ ausdrücken.

Huang konnte die Gl. (1) auf sehr einfache Weise lösen, wenn er zusätzlich die Gleichungen

$$
4 \pi \varrho=\operatorname{div} \mathfrak{D}=\operatorname{div}(\mathfrak{F}+4 \pi \mathfrak{S})=0
$$

(der Kristall ist im Mittel ungeladen)

und

$$
\operatorname{rot} \mathfrak{F}=0
$$

(Grundgleichung der Elektrostatik) berücksichtigte. Indem er nämlich $\mathfrak{w}$ in seinen quellen- und wirbelfreien Anteil, d. h. in seine transversale $\mathfrak{w}_{\mathrm{tr}}$ und longitudinale Komponente $\mathfrak{w}_{1}$ zerlegte, erhielt er für $\mathfrak{w}_{\mathrm{tr}}$ und $\mathfrak{w}_{1}$ zwei voneinander unabhängige Schwingungsgleichungen, deren zugehörige Frequenzen durch

$$
\omega_{\mathrm{tr}}=\omega_{0} \text { bzw. } \omega_{1}=\sqrt{\frac{\varepsilon_{0}}{\varepsilon_{\infty}}} \omega_{0}=\sqrt{\frac{\varepsilon_{\mathbf{0}}}{\varepsilon_{\infty}}} \omega_{\mathrm{tr}}
$$

gegeben werden. Dabei ist $\omega_{0}$ die oben erwähnte Dispersionsfrequenz. Die zweite Gleichung ist die bekannte, schon im Jahre $1941^{2}$ von Lyddane, Sachs und Teller ${ }^{5}$ auf etwas anderem Wege abgeleitete Beziehung zwischen der Frequenz der longitudinalen und transversalen optischen Schwingungen.

\section{Grundgleichungen für die langen optischen Schwingungen}

Im folgenden soll nun gezeigt werden, daß man die langen optischen Schwingungen in zweiatomigen Kristallen mit trigonaler, tetragonaler oder hexagonaler Symmetrie ebenfalls phänomenologisch behandeln kann. Für diese Kristalle lassen sich nämlich zwei Grundgleichungen in einer zu (1) analogen

was früher von Lyddane und Herzfeld $[1938]^{3}$ und FröHLICH und Motr [1939] ${ }^{4}$ gezeigt.

* Teilauszug aus der Dissertation, Münster 1959.

* Jetzt: OSRAM-Studiengesellschaft, Augsburg.

1 K. Huang, a) E. R. A. Report L/T 239 [1950]; b) Proc. Roy. Soc., Lond. A 208, 352 [1951].

$2 \mathrm{Da} \beta$ die Frequenzen der transversalen und longitudinalen langen optischen Schwingungen bei polaren kubischen Kristallen voneinander verschieden sind, wurde schon et-
3 R.H.Lyddane u. K.F. Herzfeld, Phys. Rev. 54, 846 [1938].

${ }^{4}$ H. Fröhlich u. N. F. Motт, Proc. Roy. Soc., Lond. A 171, 496 [1939].

5 R. H. Lyddane, R. G. Sachs u. E. Teller, Phys. Rev. 59, 673 [1941]. 
Form ableiten:

$$
\begin{aligned}
& \ddot{\mathfrak{w}}=B^{11} \cdot \mathfrak{w}+B^{12} \cdot \mathfrak{F}, \\
& \mathfrak{H}=B^{21} \cdot \mathfrak{w}+B^{22} \cdot \mathfrak{F} .
\end{aligned}
$$

Sie seien hier zunächst axiomatisch eingeführt und sollen in einem Anhang (s. S. 54) näher begründet werden. - Zum Unterschied von (1) sind die $B^{11}$, $B^{12}, B^{21}, B^{22}$ jetzt aber keine Skalare mehr, sondern - unter den im Anhang angegebenen Voraussetzungen - Dyaden in der speziellen Diagonalform:

$$
B=B_{1} \mathfrak{i} \mathfrak{i}+B_{2} \mathfrak{j} \mathfrak{j}+B_{3} \mathfrak{f} \text { mit } B_{1}=B_{2} \text {. }
$$

(Die $x_{3}$-Achse sei dabei die Drehachse.)

Diese Form wird durch die Symmetrie gefordert. Es gilt auch hier die Beziehung:

$$
B^{21}=B^{12} .
$$

Wenn wir für $\mathfrak{m}$, $\mathfrak{E}$ und $\mathfrak{P}$ periodische Lösungen mit dem Zeitfaktor $e^{-i \omega t}$ ansetzen, so lassen sich die Koeffizienten in der folgenden Weise aus den Dispersionsformeln bestimmen: Wir setzen $\ddot{\mathfrak{w}}=-\omega^{2} \mathfrak{w}$ in $(4 a)$ ein und eliminieren $\mathfrak{w}$ :

$$
\mathfrak{P}=\left\{B^{22}-B^{21 \cdot}\left(B^{11}+\omega^{2} I\right)^{-1} \cdot B^{12}\right\} \cdot \mathfrak{E} .
$$

Aus

$$
\mathfrak{D}=\mathfrak{E}+4 \pi \mathfrak{B}=\varepsilon \cdot \mathfrak{F},
$$

d. h.

$$
\mathfrak{P}=\frac{(\varepsilon-I)}{4 \pi} \cdot \mathfrak{F},
$$

folgt dann durch Vergleich mit (7) :

$$
\varepsilon=I+4 \pi B^{22}-4 \pi B^{21} \cdot\left(B^{11}+\omega^{2} I\right)^{-1} \cdot B^{12} .
$$

Für die Diagonalelemente von

$$
\varepsilon=\left(\begin{array}{lll}
\varepsilon_{1} & & \\
& \varepsilon_{1} & \\
& & \varepsilon_{3}
\end{array}\right)
$$

ergibt sich mithin:

$$
\begin{aligned}
& \varepsilon_{1}=1+4 \pi B_{1}^{22}-\frac{4 \pi B_{1}^{21} B_{1}^{12}}{B_{1}^{11}+\omega^{2}}, \\
& \varepsilon_{3}=1+4 \pi B_{3}^{22}-\frac{4 \pi B_{3}^{21} B_{3}^{12}}{B_{3}^{11}+\omega^{2}}
\end{aligned}
$$

oder (die Indizes 1 und 3 sind im folgenden hinzuzudenken) :

$$
\varepsilon=1+4 \pi B^{22}-\frac{4 \pi \frac{B^{21} B^{12}}{B^{11}}}{1-\left(\frac{\omega}{\sqrt{-B^{11}}}\right)^{2}},
$$

* Da die linken Seiten die Quadrate der Hauptbrechungsindizes sind, erhält man auf diese Weise allerdings nur die d. h. die Dispersionsformel * Indem wir sie mit der Dispersionsformel in der üblichen Gestalt vergleichen:

$$
\begin{aligned}
& \varepsilon=\varepsilon^{\infty}+\frac{\varepsilon^{0}-\varepsilon^{\infty}}{1-\left(\omega / \omega^{0}\right)^{2}} \\
&\left(\begin{array}{lll}
\varepsilon=\varepsilon^{0} & \text { für } \omega \rightarrow 0, & \text { d. h. } \omega \ll \omega^{0} \\
\varepsilon=\varepsilon^{\infty} \text { für } \omega \rightarrow \infty, & \text { d. h. } \omega \gg \omega^{0}
\end{array}\right),
\end{aligned}
$$

ergibt sich:

$$
\begin{aligned}
& \varepsilon^{\infty}=1+4 \pi B^{22}, \text { d. h. } B^{22}=\frac{\varepsilon^{\infty}-1}{4 \pi} ; \\
& \omega^{0}=\sqrt{-B^{11}}, \text { d. h. } B^{11}=-\left(\omega^{0}\right)^{2} ;
\end{aligned}
$$

$\varepsilon^{0}-\varepsilon^{\infty}=-4 \pi\left(B^{12}\right)^{2} / B^{11}$,

$$
\text { d. h. } B^{12}=B^{21}=\sqrt{\frac{\varepsilon^{0}-\varepsilon^{\infty}}{4 \pi}} \omega^{0} .
$$

In der letzten Gleichung wurde dabei von der Voraussetzung $B^{21}=B^{12}$ Gebrauch gemacht. In der Bezeichnung wurde von der üblichen Schreibweise $\varepsilon_{0}$, $\varepsilon_{\infty}, \omega_{0}$ durch Hochstellen der Indizes abgewichen, um den rechten unteren Platz für die noch hinzuzufügenden Indizes 1 und 3 frei zu haben.

Bevor wir die Gln. (4) zur Bestimmung der Gitterschwingungen benutzen, sei etwas über den Grad ihrer Gültigkeit gesagt: Sie sind als exakt anzusehen, sofern man in der Energiedichte (vgl. Anhang) höhere als quadratische Terme vernachlässigen darf und sich $\mathfrak{w}, \mathfrak{s}, \mathfrak{F}$ innerhalb Entfernungen von der Größenordnung der Gitterkonstanten praktisch nicht ändern. Außerdem soll die Mitbewegung der Elektronen adiabatisch erfolgen. Sie geben jedenfalls, wenn man die experimentellen Daten als hinreichend genau voraussetzt, die langen optischen Schwingungen besser wieder als irgendein mikroskopisches Modell, bei dem z. B. die Ionen als Punktladungen behandelt werden. So ist u. a. der Einfluß der Ionenpolarisation in den Gleichungen implizit enthalten, der sich bei komplizierteren Kristallen meist nur sehr schwer aus dem mikroskopischen Modell bestimmen läßt. Das Auftreten des Gliedes $B^{22}$. E beispielsweise ist allein auf Polarisationseffekte zurückzuführen.

Da die Koeffizienten in unserem Falle Dyaden sind, führt der von Huang benutzte Lösungsweg durch Zerlegen von $\mathfrak{w}$ in $\mathfrak{w}_{\mathrm{tr}}$ und $\mathfrak{m}_{1}$ nicht mehr $\mathrm{zu}$ einer Aufspaltung in zwei voneinander unabhängige Gleichungen für $\mathfrak{m}_{\mathrm{tr}}$ und $\mathfrak{m}_{1}$.

Dispersionsformeln für die ausgezeichneten Kristallrichtungen, was hier genügt. 
Wir müssen daher etwas anders vorgehen. Die drei Vektoren $\mathfrak{m}$, $\mathfrak{F}$, $\mathfrak{P}$ wären jedenfalls (bis auf einen Faktor) bestimmt, besäßen wir noch eine dritte, von (4) unabhängige Gleichung zwischen den drei Vektoren. (Die Gleichung $\mathfrak{D}=\varepsilon$. $\mathcal{E}$ ist nicht mehr unabhängig, da sie bereits zur Bestimmung der Koeffizienten $B^{i k}$ benutzt wurde.) Eine solche zusätzliche Gleichung läßt sich aber leicht gewinnen, wenn wir wieder die Gültigkeit der Gln. (2 a) und (2 b) fordern (elektrostatische Näherung). Machen wir nämlich für $\mathfrak{F}$ und $\mathfrak{s}$ den Ansatz ebener Wellen, so dürfen wir statt $\left(2\right.$ a) schreiben ${ }^{6}$ :

$$
\operatorname{div}\left(\mathfrak{E}+4 \pi \mathfrak{S}_{\|}\right)=0,
$$

da div $\mathfrak{P}_{\perp}=0$. Hierbei bedeute $\mathfrak{P}_{\|}$die Komponente

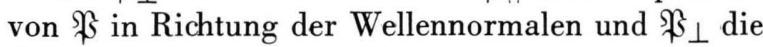
dazu senkrechte Komponente. Da außerdem

$$
\operatorname{rot} \mathfrak{\Re}_{\| 1}=0
$$

(die Rotation einer longitudinalen Schwingung verschwindet!), ergibt sich mit (2 b) :

$$
\operatorname{rot}\left(\mathfrak{F}+4 \pi \mathfrak{P}_{\|}\right)=0 .
$$

(10 a) und (10 b) zusammen verlangen aber:

oder

$$
\mathfrak{\xi}+4 \pi \mathfrak{\Re}_{\|}=0
$$

$$
\mathfrak{F}=-4 \pi \mathfrak{P}_{\|}=-4 \pi \mathfrak{n}(\mathfrak{n} \cdot \mathfrak{P})=-T \cdot \mathfrak{R} .
$$

Dabei ist $\mathfrak{n}$ der Normalen-Einheitsvektor und $T$ definiert durch

$$
T \equiv 4 \pi \mathfrak{n} .
$$

In (11) haben wir die gewünschte dritte Grundgleichung.

Für die spätere Rechnung sei $T$ noch in Komponentendarstellung explizit angegeben:

$$
T=\left(\begin{array}{lll}
T_{11} & T_{12} & T_{13} \\
T_{21} & T_{22} & T_{23} \\
T_{31} & T_{32} & T_{33}
\end{array}\right) \quad \text { mit } T_{\alpha \beta} \equiv 4 \pi n_{\alpha} n_{\beta}
$$

Offensichtlich gilt:

$$
\begin{aligned}
T_{a \beta}= & T_{\beta a} \text { (die Dyade ist symmetrisch), } \\
& T_{a \beta} T_{\gamma \delta}=T_{a \delta} T_{\gamma \beta}, \\
& T_{11}+T_{22}+T_{33}=4 \pi .
\end{aligned}
$$

\footnotetext{
${ }^{6}$ M. Born u. K. Huang, Dynamical Theory of Crystal Lattices, Clarendon Press, Oxford 1954, S. 249.
}

Wegen (12 b) verschwinden sämtliche zweireihigen Unterdeterminanten von (12), d. h. T hat den Rang eins.

\section{Berechnung der Frequenzen der Eigen- schwingungen}

Machen wir jetzt für die drei Vektoren $\mathfrak{m}$, \& und 路 den Ansatz ebener Wellen, so ergibt sich aus (4) :

$$
\begin{aligned}
-\omega^{2} \mathfrak{w} & =B^{11} \cdot \mathfrak{w}+B^{12} \cdot \mathfrak{F}, \\
\mathfrak{P} & =B^{21} \cdot \mathfrak{w}+B^{22} \cdot \mathfrak{F} .
\end{aligned}
$$

Da die gemeinsamen Phasenfaktoren sich herauskürzen lassen, können wir im weiteren unter $\mathfrak{w}, \mathfrak{F}, \mathfrak{P}$ die Amplitudenvektoren allein verstehen.

Uns interessiert zunächst die Schwingungsgleichung für $\mathfrak{w}$. Um sie zu gewinnen, setzen wir (13 b) in (11) ein, lösen nach $\mathfrak{F}$ auf:

$$
\mathcal{F}=-\left(I+T \cdot B^{22}\right)^{-1} \cdot T \cdot B^{21} \cdot \mathfrak{w}
$$

und erhalten nach Einsetzen in (13 a) :

$-\omega^{2} \mathfrak{w}=\left\{B^{11}-B^{12} \cdot\left(I+T \cdot B^{22}\right)^{-1} \cdot T \cdot B^{21}\right\} \cdot \mathfrak{w}$.

Mit der Abkürzung

$$
K \equiv B^{11}-B^{12} \cdot\left(I+T \cdot B^{22}\right)^{-1} \cdot T \cdot B^{21}
$$

können wir hierfür auch einfacher schreiben:

$$
\left(K+\omega^{2} I\right) \cdot \mathfrak{w}=0 .
$$

Durch die Schwingungsgleichung (14 a) bzw. (14 c) werden drei Eigenfrequenzen $\omega_{\alpha}(\alpha=1,2,3)$ und drei zugehörige Eigenvektoren $\mathfrak{w}_{\alpha}$ bestimmt.

Die Eigenfrequenzen ergeben sich dabei aus der zugehörigen Säkulargleichung:

$$
\left|K+\omega^{2} I\right|=0 .
$$

Ihrer Berechnung wollen wir uns jetzt zuwenden. Zunächst kann man den zweiten Term von $K$, nämlich:

$$
K_{2} \equiv B^{12} \cdot\left(I+T \cdot B^{22}\right)^{-1} \cdot T \cdot B^{21}
$$

noch wesentlich vereinfachen. Dazu benutzen wir die Determinante von $I+T \cdot B^{22}$ :

$$
D \equiv\left|I+T \cdot B^{22}\right| .
$$

Schreiben wir sie in der Form

$$
D=\frac{1}{A_{1}^{2} A_{3}} D^{\prime}
$$


mit

$$
D^{\prime}=\left|\begin{array}{ccc}
A_{1}+T_{11} & T_{19} & T_{31} \\
T_{12} & A_{1}+T_{29} & T_{23} \\
T_{31} & T_{23} & A_{3}+T_{33}
\end{array}\right|
$$

und

$$
A_{1} \equiv \mathrm{l} / B_{1}^{22}, \quad A_{3} \equiv \mathrm{l} / B_{3}^{22},
$$

so ergibt sich für $D^{\prime}$, indem man etwa nach der ersten Zeile entwickelt und (12a) und (12 b) berücksichtigt :

$$
D^{\prime}=A_{1}^{2} A_{3}+A_{1}^{2} T_{33}+A_{1} A_{3}\left(T_{11}+T_{22}\right) .
$$

Damit wird

$$
\begin{aligned}
D & =1+\frac{1}{A_{3}} T_{33}+\frac{1}{A_{1}}\left(T_{11}+T_{22}\right) \\
& =1+B_{3}^{3.2} T_{33}+B_{1}^{2 \cdot 2}\left(T_{11}+T_{22}\right)
\end{aligned}
$$

oder, wenn man $(12 \mathrm{c})$ benutzt:

$$
D=1+4 \pi B_{1}^{22}+\left(B_{3}^{22}-B_{1}^{22}\right) T_{33} .
$$

Mit Hilfe des doppeltskalaren Produktes läßt sich (16 a) auch kürzer in der Form schreiben:

$$
D=1+T \cdot B^{22} .
$$

Hiermit ergibt sich unter Beachtung der Definition von $T$ :

$$
\left(I+T \cdot B^{22}\right) \cdot T=\left(1+T \cdot B^{22}\right) T=D T
$$

und, indem man von links mit $\left(I+T \cdot B^{22}\right)^{-1}$ multipliziert und durch $D$ dividiert

$$
\left(I+T \cdot B^{22}\right)^{-1} \cdot T=\frac{1}{D} T
$$

(das Inverse von $I+T \cdot B^{22}$ existiert, da $D \neq 0$ ) . Somit erhalten wir für $K_{2}$ [Gl. (14 e) ] :

$$
\begin{aligned}
K_{2} & =\frac{1}{D} B^{12} \cdot T \cdot B^{21} \\
& =\frac{1}{D}\left(\begin{array}{lll}
\left(B_{1}^{12}\right)^{2} T_{11} & \left(B_{1}^{12}\right)^{2} T_{12} & B_{1}^{12} B_{3}^{12} T_{31} \\
\left(B_{1}^{12}\right)^{2} T_{12} & \left(B_{1}^{12}\right)^{2} T_{22} & B_{1}^{12} B_{3}^{12} T_{23} \\
B_{3}^{12} B_{1}^{12} T_{31} & B_{3}^{12} B_{1}^{12} T_{23} & \left(B_{3}^{12}\right)^{2} T_{33}
\end{array}\right)=\frac{K_{2}^{\prime}}{D} .
\end{aligned}
$$

In (19 b) wurde dabei von $B^{21}=B^{12}$ [Gl. (6) ] Gebrauch gemacht. Interessant ist an dem Ergebnis, daß das Glied $B^{22}$. F (das nur von der Ionenpolarisation herrührt) sich nur noch in dem Faktor $1 / D$ bemerkbar macht. Ohne Ionenpolarisation wäre $D=1$ [vgl. G. (16) ].

Unter Beachtung der Definition von $K_{2}^{\prime}$ in $(19 \mathrm{~b})$ läßt sich die Schwingungsgleichung $(14 \mathrm{c}) \mathrm{bzw}$. die zugehörige Säkulargleichung ( $14 \mathrm{~d}$ ) jetzt umformen in:

$$
\left\{-D\left(B^{11}+\omega^{2} I\right)+K_{2}^{\prime}\right\} \cdot \mathfrak{w}=\left\{A^{\prime}+K_{2}^{\prime}\right\} \cdot \mathfrak{w}
$$

bzw.

$$
\left|-D\left(B^{11}+\omega^{2} I\right)+K_{2}^{\prime}\right|=\left|A^{\prime}+K_{2}^{\prime}\right|=0,
$$

wobei

$$
A^{\prime} \equiv-D\left(B^{11}+\omega^{2} I\right)
$$

Diagonalgestalt hat. Führen wir außerdem die Abkürzungen

$T_{\alpha \beta}^{\prime}=B_{\alpha}^{12} B_{\beta}^{12} T_{\alpha \beta}\left(\operatorname{mit} B_{.2}^{12}=B_{1}^{12}\right) \quad(\alpha, \beta=1,2,3)$

ein, so hat die Determinante in $(20 \mathrm{~b})$ genau die Gestalt von $D^{\prime}$ [Gl. (15 c) ] :

$$
\left|\begin{array}{ccc}
A_{1}^{\prime}+T_{11}^{\prime} & T_{12}^{\prime} & T_{31}^{\prime} \\
T_{12}^{\prime} & A_{1}^{\prime}+T_{22}^{\prime} & T_{23}^{\prime} \\
T_{31}^{\prime} & T_{23}^{\prime} & A_{3}^{\prime}+T_{33}^{\prime}
\end{array}\right|=0 .
$$

Die Ausrechnung ergibt wie dort:

$$
A_{1}^{\prime 2} A_{3}^{\prime}+A_{1}^{\prime 2} T_{33}^{\prime}+A_{1}^{\prime} A_{3}^{\prime}\left(T_{11}^{\prime}+T_{22}^{\prime}\right)=0,
$$

woraus man als erste Lösung abliest :

$A_{1}^{\prime}=-D\left(B_{1}^{11}+\omega^{2} I\right)=0$,

$$
\begin{gathered}
\omega_{1}^{2}=-B_{1}^{11}, \\
\omega_{1}=\sqrt{-B_{1}^{11}}=\omega_{1}^{0} \text { nach Gl. (9). }
\end{gathered}
$$

Eine Eigenfrequenz stimmt also überein mit einer Dispersionsfrequenz, nämlich der zweifachen $\omega_{1}^{0}$.

Durch den Restfaktor in ( $22 \mathrm{~b})$ werden die zwei weiteren Eigenfrequenzen bestimmt:

$$
\begin{gathered}
A_{1}^{\prime} A_{3}^{\prime}+A_{1}^{\prime} T_{33}^{\prime}+A_{3}^{\prime}\left(T_{11}^{\prime}+T_{22}^{\prime}\right)=0, \quad(24 \mathrm{a}) \\
D\left(B_{1}^{11}+\omega^{2}\right)\left(B_{3}^{11}+\omega^{2}\right)-\left(B_{1}^{11}+\omega^{2}\right) T_{33}^{\prime} \quad(24 \mathrm{~b}) \\
-\left(B_{3}^{11}+\omega^{2}\right)\left(T_{11}^{\prime}+T_{22}^{\prime}\right)=0, \\
D \omega^{4}-\left[-D\left(B_{1}^{11}+B_{3}^{11}\right)+T_{11}^{\prime}+T_{22}^{\prime}+T_{33}^{\prime}\right] \omega^{2} \\
+\left[D B_{1}^{11} B_{3}^{11}-B_{1}^{11} T_{33}^{\prime}-B_{3}^{11}\left(T_{11}^{\prime}+T_{22}^{\prime}\right)\right]=0 .
\end{gathered}
$$

Führen wir für die eckigen Klammern die Abkürzung $K$ bzw. $M$ ein, so läßt sich die Gleichung in der Form schreiben:

$$
\omega^{4}-\frac{K}{D} \omega^{2}+\frac{M}{D}=0 .
$$

Sie hat die beiden Lösungen:

$$
\omega_{2,3}^{2}=\frac{1}{2 D}\left[K \mp \sqrt{ } K^{2}-4 D M\right],
$$


wobei der Index 2 sich auf das negative, der Index 3 auf das positive Wurzelvorzeichen bezieht.

Die Ausdrücke für $K$ und $M$ lassen sich noch etwas übersichtlicher schreiben. Man kann sie näm- lich unter Beachtung von (21), (12 c) und (16b) in einen richtungsunabhängigen und einen richtungsabhängigen Term aufspalten (letzterer enthält $T_{33}$ als Faktor) :

$$
\begin{aligned}
K & =-D\left(B_{1}^{11}+B_{3}^{11}\right)+\left(B_{1}^{12}\right)^{2}\left(T_{11}+T_{22}\right)+\left(B_{3}^{12}\right)^{2} T_{33}=-D\left(B_{1}^{11}+B_{3}^{11}\right)+4 \pi\left(B_{1}^{12}\right)^{2}+\left[\left(B_{3}^{12}\right)^{2}-\left(B_{1}^{12}\right)^{2}\right] T_{33} \\
& =-\left(1+4 \pi B_{1}^{22}\right)\left(B_{1}^{11}+B_{3}^{11}\right)+4 \pi\left(B_{1}^{12}\right)^{2}+\left[\left(B_{1}^{22}-B_{3}^{22}\right)\left(B_{1}^{11}+B_{3}^{11}\right)+\left(B_{3}^{12}\right)^{2}-\left(B_{1}^{12}\right)^{2}\right] T_{33},
\end{aligned}
$$

$M=D B_{1}^{11} B_{3}^{11}-B_{1}^{11}\left(B_{3}^{12}\right)^{2} T_{33}-\left(B_{1}^{12}\right)^{2} B_{3}^{11}\left(T_{11}+T_{22}\right)=D B_{1}^{11} B_{3}^{11}-4 \pi\left(B_{1}^{12}\right)^{2} B_{3}^{11}+\left[\left(B_{1}^{12}\right)^{2} B_{3}^{11}-B_{1}^{11}\left(B_{3}^{12}\right)^{2}\right] T_{33}$

$$
=\left(1+4 \pi B_{1}^{22}\right) B_{1}^{11} B_{3}^{11}-4 \pi\left(B_{1}^{12}\right)^{2} B_{3}^{11}+\left[\left(B_{3}^{22}-B_{1}^{22}\right) B_{1}^{11} B_{3}^{11}+\left(B_{1}^{12}\right)^{2} B_{3}^{11}-B_{1}^{11}\left(B_{3}^{12}\right)^{2}\right] T_{33} .
$$

Für eine Anwendung der Gleichungen interessiert besonders, in welcher Weise sich $D, K$ und $M$ durch die Dielektrizitätskonstanten und die Dispersionsfrequenzen ausdrücken lassen. Durch Einsetzen von (9) in (16 b), (26 a) und (26 b) ergibt sich aber sofort (man beachte $T_{33}=4 \pi n_{3}^{2}[\mathrm{vgl}$. (12) ]) :

$D=\varepsilon_{1}^{\infty}+\left[\varepsilon_{3}^{\infty}-\varepsilon_{1}^{\infty}\right] n_{3}^{2}$,

$$
\begin{aligned}
K & =\varepsilon_{1}^{\infty}\left(\left(\omega_{1}^{0}\right)^{2}+\left(\omega_{3}^{0}\right)^{2}\right)+\left(\varepsilon_{1}^{0}-\varepsilon_{1}^{\infty}\right)\left(\omega_{1}^{0}\right)^{2}+\left[\left(\varepsilon_{3}^{\infty}-\varepsilon_{1}^{\infty}\right)\left(\left(\omega_{1}^{0}\right)^{2}+\left(\omega_{3}^{0}\right)^{2}\right)+\left(\varepsilon_{1}^{\infty}-\varepsilon_{1}^{0}\right)\left(\omega_{1}^{0}\right)^{2}+\left(\varepsilon_{3}^{0}-\varepsilon_{3}^{\infty}\right)\left(\omega_{3}^{0}\right)^{2}\right] n_{3}^{2} \\
& =\left(\varepsilon_{1}^{0}\left(\omega_{1}^{0}\right)^{2}+\varepsilon_{1}^{\infty}\left(\omega_{3}^{0}\right)^{2}\right)+\left[\left(\varepsilon_{3}^{\infty}-\varepsilon_{1}^{0}\right)\left(\omega_{1}^{0}\right)^{2}+\left(\varepsilon_{3}^{0}-\varepsilon_{1}^{\infty}\right)\left(\omega_{3}^{0}\right)^{2}\right] n_{3}^{2},
\end{aligned}
$$

$$
\begin{aligned}
M & =\varepsilon_{1}^{\infty}\left(\omega_{1}^{0}\right)^{2}\left(\omega_{3}^{0}\right)^{2}+\left(\varepsilon_{1}^{0}-\varepsilon_{1}^{\infty}\right)\left(\omega_{1}^{0}\right)^{2}\left(\omega_{3}^{0}\right)^{2}+\left[\left(\varepsilon_{3}^{\infty}-\varepsilon_{1}^{\infty}\right)\left(\omega_{1}^{0}\right)^{2}\left(\omega_{3}^{0}\right)^{2}-\left(\varepsilon_{1}^{0}-\varepsilon_{1}^{\infty}\right)\left(\omega_{1}^{0}\right)^{2}\left(\omega_{3}^{0}\right)^{2}+\left(\varepsilon_{3}^{0}-\varepsilon_{3}^{\infty}\right)\left(\omega_{1}^{0}\right)^{2}\left(\omega_{3}^{0}\right)^{2}\right] n_{3}^{2} \\
& =\left(\omega_{1}^{0}\right)^{2}\left(\omega_{3}^{0}\right)^{2}\left(\varepsilon_{1}^{0}+\left[\varepsilon_{3}^{0}-\varepsilon_{1}^{0}\right] n_{3}^{2}\right) .
\end{aligned}
$$

Es sei darauf verzichtet, diese Ausdrücke in (25) explizit einzusetzen, da (25) dann wenig übersichtlich würde. Wir wollen vielmehr die Formeln (23) bzw. (25) in Verbindung mit (26) und (27) noch diskutieren:

Zunächst sieht man: Von den drei Frequenzen der optischen Eigenschwingungen ist nur eine (nämlich $\omega_{1}[\mathrm{Gl} .(23)]$ ), wie bereits erwähnt, gleich einer Dispersionsfrequenz und damit auch richtungsunab- hängig. Die beiden anderen nehmen dagegen die Dispersionsfrequenzen höchstens in Spezialfällen an und sind wegen des Faktors $n_{3}^{2}$ richtungsabhängig.

Wir erhalten einen besseren Überblick, wenn wir einige Spezialfälle betrachten.

1. Wir lassen $\omega_{3}^{0} \rightarrow \omega_{1}^{0}, \varepsilon_{3}^{0} \rightarrow \varepsilon_{1}^{0}, \varepsilon_{3}^{\infty} \rightarrow \varepsilon_{1}^{\infty}$ gehen, d. h. gehen zur kubischen Symmetrie über. Dann ergibt sich:

$$
\begin{aligned}
& D \rightarrow \varepsilon_{1}^{\infty}, \\
& K \rightarrow\left(\varepsilon_{1}^{0}+\varepsilon_{1}^{\infty}\right)\left(\omega_{1}^{0}\right)^{2}, \\
& M \rightarrow\left(\omega_{1}^{0}\right)^{4} \varepsilon_{1}^{0}, \\
& \sqrt{K^{2}-4 D M} \rightarrow\left(\omega_{1}^{0}\right)^{2}\left(\varepsilon_{1}^{0}-\varepsilon_{1}^{\infty}\right),
\end{aligned}
$$

$$
\begin{aligned}
& \omega_{2,3}^{2} \rightarrow \frac{\left(\omega_{1}^{0}\right)^{2}}{2 \varepsilon_{1}^{\infty}}\left[\varepsilon_{1}^{0}+\varepsilon_{1}^{\infty} \mp\left(\varepsilon_{1}^{0}-\varepsilon_{1}^{\infty}\right)\right]: \\
& \omega_{2}^{2} \rightarrow\left(\omega_{1}^{0}\right)^{2} \text { oder } \quad \underline{\omega_{2} \rightarrow \omega_{1}^{0}} \\
& \omega_{3}^{2} \rightarrow \frac{\varepsilon_{1}^{0}}{\varepsilon_{1}^{\infty}}\left(\omega_{1}^{0}\right)^{2} \text { oder } \omega_{3} \rightarrow \sqrt{\frac{\varepsilon_{1}^{0}}{\varepsilon_{1}^{\infty}}} \omega_{1}^{0} .
\end{aligned}
$$

Ersetzt man in $(28) \rightarrow$ durch $=$, so hat man zusammen mit (23) die bekannten Formeln für die Frequenzen der langen optischen Schwingungen in kubischen Kristallen (vgl. Anm. ${ }^{5}$ ). Sie sind im Gegensatz zu (25) alle richtungsunabhängig und nur eine von ihnen (nämlich $\omega_{3}=\omega_{1}$, d. i. die Frequenz der longitudinalen Schwingungen) von der Dispersionsfrequenz verschieden.

2. Wir betrachten die Schwingungen mit dem Normalenvektor

a) senkrecht zur Drehachse,

b) in Richtung der Drehachse. 
Im Falle a) ist $n_{3}=0$. Damit folgt aus (27) und (25):

$$
\begin{array}{lc}
D=\varepsilon_{1}^{\infty}, & \omega_{2,3}^{2}=\frac{1}{2 \varepsilon_{1}^{\infty}}\left[\varepsilon_{1}^{0}\left(\omega_{1}^{0}\right)^{2}+\varepsilon_{1}^{\infty}\left(\omega_{3}^{0}\right)^{2} \mp\left(\varepsilon_{1}^{0}\left(\omega_{1}^{0}\right)^{2}-\varepsilon_{1}^{\infty}\left(\omega_{3}^{0}\right)^{2}\right)\right]: \\
K=\varepsilon_{1}^{0}\left(\omega_{1}^{0}\right)^{2}+\varepsilon_{3}^{0}\left(\omega_{3}^{0}\right)^{2}, & \omega_{2}^{2}=\left(\omega_{3}^{0}\right)^{2}, \quad l \quad l \\
M=\left(\omega_{1}^{0}\right)^{2}\left(\omega_{3}^{0}\right)^{2} \varepsilon_{1}^{0}, & \omega_{3}^{2}=\frac{\omega_{1}^{0}}{\varepsilon_{1}^{\infty}}\left(\omega_{1}^{0}\right)^{2}, \quad \omega_{3}=\sqrt{\frac{\varepsilon_{1}^{0}}{\varepsilon_{1}^{\infty}}} \omega_{1}^{0} . \\
\sqrt{K^{2}-4 M D}=\varepsilon_{1}^{0} \omega_{1}^{0}-\varepsilon_{1}^{\infty} \omega_{3}^{0} \quad \text { (s. Anm. }{ }^{*} \text { ). }
\end{array}
$$

Im Falle b) ist $n_{3}=1$. Damit folgt :

$$
\begin{array}{lll}
D=\varepsilon_{3}^{\infty}, & \omega_{2,3}^{2}=\frac{1}{2 \varepsilon_{3}^{\infty}}\left[\varepsilon_{3}^{\infty}\left(\omega_{1}^{0}\right)^{2}+\varepsilon_{3}^{0}\left(\omega_{3}^{0}\right)^{2} \mp\left(\varepsilon_{3}^{0}\left(\omega_{3}^{0}\right)^{2}-\varepsilon_{3}^{\infty}\left(\omega_{1}^{0}\right)^{2}\right)\right]: \\
K=\varepsilon_{3}^{\infty}\left(\omega_{1}^{0}\right)^{2}+\varepsilon_{3}^{0}\left(\omega_{3}^{0}\right)^{2}, & \omega_{2}^{2}=\left(\omega_{1}^{0}\right)^{2}, & \frac{\omega_{2}=\omega_{1}^{0},}{\omega_{3},} \\
M=\left(\omega_{1}^{0}\right)^{2}\left(\omega_{3}^{0}\right)^{2} \varepsilon_{3}^{0}, & \omega_{3}^{2}=\frac{\varepsilon_{3}^{0}}{\varepsilon_{3}^{\infty}}\left(\omega_{3}^{0}\right)^{2}, \quad & \omega_{3}=\sqrt{\frac{\varepsilon_{3}^{0}}{\varepsilon_{3}^{\infty}}} \omega_{3}^{0} . \\
V \overline{K^{2}-4 M D}=\varepsilon_{3}^{0}\left(\omega_{3}^{0}\right)^{2}-\varepsilon_{3}^{\infty}\left(\omega_{1}^{0}\right)^{2} & \text { (s. Anm. } \left.{ }^{* *}\right) . &
\end{array}
$$

Die Formeln (29a) und (29 b) unterscheiden sich äußerlich nur dadurch von (28), daß jetzt auf der rechten Seite von (29a) bzw. (29 b) auch entsprechende Ausdrücke mit dem Index 3 auftreten.

\section{Gestalt der Polarisationsvektoren für die Eigenschwingungen}

In diesem Abschnitt soll die Frage nach der Polarisation der Eigenschwingungen behandelt werden. Die Polarisationsvektoren $\mathfrak{w}$ ergeben sich bekanntlich aus der Koeffizientenmatrix der Schwingungsgleichung $(20 \mathrm{a})$ :

$$
A^{\prime}+K_{2}^{\prime}=\left(\begin{array}{ccc}
A_{1}^{\prime}+T_{11}^{\prime} & T_{12}^{\prime} & T_{31}^{\prime} \\
T_{12}^{\prime} & A_{1}^{\prime}+T_{22}^{\prime} & T_{23}^{\prime} \\
T_{31}^{\prime} & T_{23}^{\prime} & A_{3}^{\prime}+T_{33}^{\prime}
\end{array}\right),
$$

indem man die algebraischen Komplemente zu den Elementen einer Zeile bildet (Cramersche Regel). Wählen wir zur Berechnung die erste Zeile, so folgt:

$w_{1}=\left|\begin{array}{cc}A_{1}^{\prime}+T_{22}^{\prime} & T_{23}^{\prime} \\ T_{23}^{\prime} & A_{3}^{\prime}+T_{33}^{\prime}\end{array}\right|=A_{1}^{\prime} A_{3}^{\prime}+A_{1}^{\prime} T_{33}^{\prime}+A_{3}^{\prime} T_{22}^{\prime}$,

$w_{2}=-\left|\begin{array}{cc}T_{12}^{\prime} & T_{2 \cdot}^{\prime} \\ T_{31}^{\prime} & A_{3}^{\prime}+T_{33}^{\prime}\end{array}\right|=-A_{3}^{\prime} T_{12}^{\prime}$,

$w_{3}=\left|\begin{array}{cc}T_{12}^{\prime} & A_{1}^{\prime}+T_{22}^{\prime} \\ T_{31}^{\prime} & T_{23}^{\prime}\end{array}\right|=-A_{1}^{\prime} T_{31}^{\prime}$,

oder zusammengefaßt:

$$
\mathfrak{w}=\left(A_{1}^{\prime} A_{3}^{\prime}+A_{1}^{\prime} T_{33}^{\prime}+A_{3}^{\prime} T_{22}^{\prime},-A_{3}^{\prime} T_{12}^{\prime},-A_{1}^{\prime} T_{31}^{\prime}\right) .
$$

Für die weitere Ausrechnung von (32) ist es zweckmäßig, an dieser Stelle schon eine Fallunterscheidung zu machen.

a) Es liege die Frequenz $\omega_{1}=\omega_{1}^{0}$ vor. Dann gilt [vgl. Gl. (23) ] :

$$
A_{1}^{\prime}=0 \text {. }
$$

Aus (32) folgt daher

$$
\begin{aligned}
\mathfrak{w}_{1} & =\left(A_{3}^{\prime} T_{22}^{\prime},-A_{3}^{\prime} T_{12}^{\prime}, 0\right) \\
& =f\left(n_{2},-n_{1}, 0\right) \quad \text { mit } f=4 \pi n_{2}\left(B_{1}^{12}\right)^{2} A_{3}^{\prime}
\end{aligned}
$$

[man beachte dabei (21) und (12)].

Da $\mathfrak{m}_{1}$ ohnehin nur bis auf einen willkürlichen Faktor bestimmt ist, wollen wir im folgenden unter $f$ eine beliebige Konstante verstehen.

Wegen $\mathfrak{n} \cdot \mathfrak{w}_{1}=f\left(n_{1} n_{2}-n_{2} n_{1}\right)=0$

* Damit der angegebene Ausdruck die positive Wurzel ist, muß $\frac{\varepsilon_{1}^{0}}{\varepsilon_{1}^{\infty}} \omega_{1}^{0}>\omega_{3}^{0}$, d. h. die Frequenz der longitudinalen Schwingungen größer sein als die der transversalen (vgl. hierzu weiter unten). Dies ist bei relativ kleiner Anisotropie und stärkeren Cоuцомв-Kräften auch stets der Fall. Sollte jedoch der umgekehrte Fall vorliegen, so wären die Bezeichnungen $\omega_{2}$ und $\omega_{3}$ zu vertauschen. Gl. (29 a) lautet dann:

$$
\omega_{2}=\sqrt{\frac{\overline{\varepsilon_{1}^{0}}}{\varepsilon_{1}^{\infty}}} \omega_{1}^{0} \quad(\alpha), \quad \omega_{3}=\omega_{3}^{0} \quad(\beta) .
$$

** Hier gilt Entsprechendes wie unter Anm. *. 
ist die Schwingung transversal, wobei der Polarisationsvektor aber immer fest in der Ebene senkrecht zur Drehachse liegt.

b) Es liege eine der Frequenzen $\omega_{2}$ oder $\omega_{3}$ vor [Gl. (25) ]. Da für diese Frequenzen Gl. (24 a) gilt, kann man z. B. die erste Komponente von $\mathfrak{m}_{2,3}$ in Gl. (32) umschreiben in:

$$
\begin{aligned}
A_{1}^{\prime} A_{3}^{\prime}+ & A_{1}^{\prime} T_{33}+A_{3}^{\prime} T_{22} \\
& =\left[A_{1}^{\prime} A_{3}^{\prime}+A_{1}^{\prime} T_{33}^{\prime}+A_{3}^{\prime}\left(T_{11}^{\prime}+T_{22}^{\prime}\right)\right]-A_{3}^{\prime} T_{11}^{\prime} \\
& =-A_{3}^{\prime} T_{11}^{\prime},
\end{aligned}
$$

so $\mathrm{da} ß$

$$
\mathfrak{w}_{2,3}=\left(-A_{3}^{\prime} T_{11}^{\prime},-A_{3}^{\prime} T_{12}^{\prime},-A_{1}^{\prime} T_{31}^{\prime}\right) .
$$

Um den Faktor $A_{3}^{\prime}$ vorziehen zu können, sei auch die dritte Komponente mit Hilfe von (24 a) umgeformt. Es gilt nämlich:

$$
\begin{aligned}
& -A_{1}^{\prime} T_{31}^{\prime}=-\frac{n_{1} B_{1}^{12}}{n_{3} B_{3}^{12}} A_{1}^{\prime} T_{33}^{\prime} \\
& =\frac{n_{1} B_{1}^{12}}{n_{3} B_{3}^{12}}\left[A_{1}^{\prime} A_{3}^{\prime}+A_{3}^{\prime}\left(T_{11}^{\prime}+T_{22}^{\prime}\right)\right] .
\end{aligned}
$$

Wir können somit statt (35 a) schreiben:

$$
\begin{gathered}
\mathfrak{m}_{2,3}=f\left(-4 \pi B_{1}^{12} B_{3}^{12} n_{1} n_{3},-4 \pi B_{1}^{12} B_{3}^{12} n_{2} n_{3},\right. \\
\left.A_{1}^{\prime}+4 \pi\left(B_{1}^{12}\right)^{2}\left(n_{1}^{2}+n_{2}^{2}\right)\right) \\
\text { mit } f=\frac{B_{1}^{12} n_{1}}{B_{3}^{12} n_{3}} A_{3}^{\prime} .
\end{gathered}
$$

Da $\mathfrak{m}_{2,3}$ sowieso nur bis auf einen Faktor bestimmt ist, dürfen wir im folgenden $f$ wieder als willkürlich ansehen.

Für eine weitere Auswertung wollen wir alle von $\varepsilon^{0}, \varepsilon^{\infty}, \omega^{0}$ abhängigen Faktoren der ersten und zweiten Komponente herausziehen. Indem wir dann nämlich statt $(35 \mathrm{~b})$ schreiben:

$\mathfrak{w}_{2,3}=f^{\prime}\left(-n_{1} n_{3},-n_{2} n_{3}, w_{3}^{\prime}\right)$ mit $f^{\prime} \equiv 4 \pi B_{1}^{12} B_{3}^{12} f$

und $\quad w_{3}^{\prime}=\frac{A_{1}^{\prime}}{4 \pi B_{1}^{12} B_{3}^{12}}+\frac{B_{1}^{12}}{B_{3}^{12}}\left(n_{1}^{2}+n_{2}^{2}\right)$,

brauchen wir uns im weiteren nur noch mit der dritten Komponente näher zu beschäftigen. Zunächst ergibt sich, wenn wir der Reihe nach $(20 \mathrm{c})$, (9), (27 a) einsetzen und $n_{1}^{2}+n_{2}^{2}=1-n_{3}^{2}$ beachten:

$$
\begin{aligned}
& w_{3}^{\prime}=\frac{-D\left(B_{1}^{11}+\omega_{2,3}^{2}\right)}{4 \pi B_{1}^{12} B_{3}^{12}}+\frac{B_{1}^{12}}{B_{3}^{12}}\left(n_{1}^{2}+n_{3}^{2}\right) \\
& =\frac{-D\left(-\left(\omega_{1}^{0}\right)^{2}+\omega_{2,3}^{2}\right)}{\sqrt{\left(\varepsilon_{1}^{0}-\varepsilon_{1}^{\infty}\right)\left(\varepsilon_{3}^{0}-\varepsilon_{3}^{\infty}\right)} \omega_{1}^{0} \omega_{3}^{0}}+\frac{\sqrt{\varepsilon_{1}^{0}-\varepsilon_{1}^{\infty}}}{\sqrt{\varepsilon_{3}^{0}-\varepsilon_{3}^{\infty}}} \frac{\omega_{1}^{0}}{\omega_{3}^{0}}\left(n_{1}^{2}+n_{2}^{2}\right) \\
& (36 \mathrm{~b})
\end{aligned}
$$

$$
\begin{aligned}
& =\frac{1}{a}\left\{\left(\omega_{1}^{0}\right)^{2}\left(D+\left(\varepsilon_{1}^{0}-\varepsilon_{1}^{\infty}\right)\left(n_{1}^{2}+n_{2}^{2}\right)\right)-\omega_{2,3}^{2} D\right\}(36 \mathrm{c}) \\
& =-\frac{1}{a}\left\{\left(\omega_{1}^{0}\right)^{2}\left(\varepsilon_{1}^{0}+\left(\varepsilon_{3}^{\infty}-\varepsilon_{1}^{0}\right) n_{3}^{2}\right)-\omega_{2,3}^{2} D\right\} \quad(36 \mathrm{~d})
\end{aligned}
$$

$$
\text { mit } \quad a=\sqrt{\left(\varepsilon_{1}^{0}-\varepsilon_{1}^{\infty}\right)\left(\varepsilon_{3}^{0}-\varepsilon_{3}^{\infty}\right)} \omega_{1}^{0} \omega_{3}^{0},(36 \mathrm{e})
$$

oder, wenn man noch $\omega_{2,3}^{2}$ [Gl. (25) ] einsetzt:

$$
\begin{array}{r}
w_{3}^{\prime}=\frac{1}{a}\left\{\left(\omega_{1}^{0}\right)^{2}\left(\varepsilon_{1}^{0}+\left(\varepsilon_{1}^{\infty}-\varepsilon_{1}^{0}\right) n_{3}^{2}\right) \quad(36 \mathrm{f})\right. \\
\left.-\frac{1}{2}\left(K \mp \sqrt{K^{2}-4 D M}\right)\right\} .
\end{array}
$$

$\mathfrak{w}_{2}$ ist der Eigenvektor zur Eigenfrequenz $\omega_{2}$ [negatives Wurzelvorzeichen in der eckigen Klammer von (36f) $], \mathfrak{m}_{3}$ der Eigenvektor zur Eigenfrequenz $\omega_{3}$ [positives Wurzelvorzeichen in $(36 \mathrm{f})]$. Im allgemeinen ist keiner der beiden Eigenvektoren rein transversal noch rein longitudinal. Wir können aber zeigen: Sind $\varepsilon_{3}^{0}, \varepsilon_{3}^{\infty}$ und $\omega_{3}^{0}$ nicht allzusehr von $\varepsilon_{1}^{0}, \varepsilon_{1}^{\infty}$ bzw. $\omega_{1}^{0}$ verschieden, so ist $\mathfrak{m}_{2}$ angenähert transversal, $\mathfrak{m}_{3}$ angenähert longitudinal. Wir lassen also $\varepsilon_{3}^{0} \rightarrow \varepsilon_{1}^{0}, \quad \varepsilon_{3}^{\infty} \rightarrow \varepsilon_{1}^{\infty}, \omega_{3}^{0} \rightarrow \omega_{1}^{0}$ gehen und benutzen dabei die Ergebnisse (28). Für $\mathfrak{w}_{2}$ (mit der Eigenfrequenz $\omega_{2}$ ) verschwindet der erste Term in (36 b) $\left(\omega_{2}=\omega_{1}^{0}\right)$, und es bleibt

$$
w_{3}^{\prime}=n_{1}^{2}+n_{2}^{2},
$$

d. h.

Aus

$\mathfrak{n} \cdot \mathfrak{m}_{2}=f^{\prime}\left(-n_{1}^{2} n_{3}-n_{2}^{2} n_{3}+n_{3}\left(n_{1}^{2}+n_{2}^{2}\right)\right)=0$

erkennt man, daß die Schwingung transversal ist.

Zur Berechnung von $\mathfrak{w}_{3}$ (mit der Eigenfrequenz $\left.\omega_{3}\right)$ gehen wir von $(36 \mathrm{~d})$ aus. Wegen [vgl. (28) ]

$$
\omega_{3}^{2}=\frac{\varepsilon_{1}^{0}}{\varepsilon_{1}^{\infty}}\left(\omega_{1}^{0}\right)^{2}, D=\varepsilon_{1}^{\infty}, a=\left(\varepsilon_{1}^{0}-\varepsilon_{1}^{\infty}\right)\left(\omega_{1}^{0}\right)^{2}
$$

ergibt sich

$$
w_{3}^{\prime}=-n_{3}^{2} \text {. }
$$

Folglich gilt:

$\mathfrak{m}_{3}=f^{\prime}\left(-n_{1} n_{3},-n_{2} n_{3},-n_{3}^{2}\right)=-f^{\prime} n_{3} \mathfrak{n}$,

d. h. die Schwingung ist longitudinal, was wir zeigen wollten.

Schließlich seien auch noch die Polarisationsvektoren für die zwei ausgezeichneten Richtungen des Normalenvektors, nämlich

a) senkrecht zur Drehachse,

b) in Richtung der Drehachse

berechnet, d.h. die Polarisationsvektoren zu den Frequenzen (29a) bzw. (29 b).

Fall a): Normalenvektor senkrecht zur Drehachse, $n_{3}=0$. 


$$
\text { a) } \omega_{2}=\omega_{3}^{0} \text {, Gl. }(29 \mathrm{a}, \alpha) \text {. }
$$

Aus (36) und (36 d) folgt

$$
\mathrm{m}_{2}=f^{\prime}\left(0,0, w_{3}^{\prime}\right)
$$

mit (man beachte $\left.D=\varepsilon_{1}^{\infty}\right)$ :

$$
w_{3}^{\prime}=\frac{1}{a}\left[\left(\omega_{1}^{0}\right)^{2} \varepsilon_{1}^{0}-\left(\omega_{3}^{0}\right)^{2} \varepsilon_{1}^{\infty}\right] \neq 0 .
$$

Die Schwingung erfolgt also in Richtung der Drehachse, d. h. ist transversal.

$$
\text { ק) } \omega_{3}=\sqrt{\frac{\varepsilon_{1}^{0}}{\varepsilon_{1}^{\infty}}} \omega_{1}^{0}, \quad \text { Gl. }(29 \mathrm{a}, \beta) .
$$

Wir schreiben Gl. (36) zunächst in der Form:

$$
\mathfrak{m}_{3}=f^{\prime \prime}\left(n_{1}, n_{2},-\frac{w_{3}^{\prime}}{n_{3}}\right) \quad\left(f^{\prime \prime} \equiv-n_{3} f^{\prime}\right) .
$$

Durch Einsetzen von $(29 \mathrm{a}, \beta)$ in $(36 \mathrm{~d})$ folgt nun:

$$
\frac{w_{3}^{\prime}}{n_{3}}=\frac{\left(\omega_{1}^{0}\right)^{2}}{n_{3} a}\left\{\left(\varepsilon_{3}^{\infty}-\varepsilon_{1}^{0}\right) n_{3}^{2}\right\}=\left(\omega_{1}^{0}\right)^{2} \frac{\varepsilon_{3}^{\infty}-\varepsilon_{1}^{0}}{a} n_{3}=0 \text {, }
$$

d. h.

$$
\mathfrak{m}_{3}=f^{\prime \prime}\left(n_{1}, n_{2}, 0\right) \text {. }
$$

Die Schwingung ist also longitudinal. - Nehmen wir noch (33) hinzu:

$$
\mathfrak{m}_{1}=f\left(n_{2},-n_{1}, 0\right),
$$

so haben wir also zwei transversale Schwingungen mit den Frequenzen $\omega_{1}=\omega_{1}^{0}$ bzw. $\omega_{2}=\omega_{3}^{0}$ und eine longitudinale mit der Frequenz $\omega_{3}=\sqrt{\frac{\varepsilon_{1}^{0}}{\varepsilon_{1}^{\infty}}} \omega_{1}^{0}$.

Fall $b$ ): Normalenvektor in Richtung der Drehachse, $n_{3}=1 \quad\left(n_{1}=0, n_{2}=0\right)$.

a) $\omega_{2}=\omega_{1}^{0}$, Gl. $(29 \mathrm{~b}, \alpha)$.

Wir versuchen den Schwingungsvektor durch Grenzübergang zu bestimmen. Bei der dritten Komponente des Schwingungsvektors (36) bleibt nach Einsetzen von $(29 \mathrm{~b}, \alpha)$ in $(36 \mathrm{~b})$ nur das zweite Glied stehen:

$$
w_{3}^{\prime}=\frac{\sqrt{\varepsilon_{1}^{0}-\varepsilon_{1}^{\infty}}}{\sqrt{\varepsilon_{3}^{0}-\varepsilon_{3}^{\infty}}} \frac{\omega_{1}^{0}}{\omega_{3}^{0}}\left(n_{1}^{2}+n_{2}^{2}\right)=c\left(n_{1}^{2}+n_{2}^{2}\right) .
$$

Lassen wir jetzt $n_{3} \rightarrow 1$ gehen, so nimmt (36) die Gestalt an:

$$
\mathfrak{m}_{2}=f^{\prime}\left(n_{1}, n_{2}, c\left(n_{1}^{2}+n_{2}^{2}\right)\right) .
$$

Wir müssen aber gleichzeitig $n_{1} \rightarrow 0, n_{2} \rightarrow 0$ gehen lassen. Dabei darf man für genügend kleine $n_{1}, n_{2}$ die Quadrate $n_{1}^{2}, n_{2}^{2}$ gegenüber $n_{1}, n_{2}$ vernachlässigen, d. h. die dritte Komponente in (43) gleich Null setzen. Beim weiteren Grenzübergang haben wir zu beachten, daß der Polarisationsvektor $\mathfrak{m}_{2}$ als normiert anzusehen ist (bei festem $f^{\prime}$ würde man beim Grenzübergang immer den Nullvektor erhalten), d. h. wir schreiben:

$$
\mathfrak{m}_{2}=\frac{1}{\sqrt{n_{1}^{2}+n_{2}^{2}}}\left(n_{1}, n_{2}, 0\right) .
$$

Dieser Form sieht man aber ohne weiteres an, da $\beta$ der Grenzvektor für $n_{1} \rightarrow 0, n_{2} \rightarrow 0$ nicht eindeutig ist. Es läßt sich vielmehr jeder vorgegebene Vektor

$$
\mathrm{m}_{2}=(a, b, 0) \quad \text { mit } a^{2}+b^{2}=1
$$

erhalten, wenn man nur $n_{1}, n_{2}$ im Verhältnis $n_{1} / n_{2}=a / b$ gegen Null gehen läßt. Wir haben somit gefunden: Die Schwingung ist transversal, wobei der Polarisationsvektor aber in der Ebene senkrecht zur Drehachse nicht festliegt. - Das gleiche gilt für den Schwingungsvektor $\mathrm{m}_{1}[$ Gl. (33) ].

$$
\text { в) } \omega_{3}=\sqrt{\varepsilon_{3}^{0} / \varepsilon_{3}^{\infty} \omega_{3}^{0}}, \quad \text { Gl. }(29 \mathrm{~b}, \beta) .
$$

Durch Einsetzen von $(29 \mathrm{~b}, \beta)$ und $n_{3}=1$ in $(36 \mathrm{~d})$ folgt $\left(D=\varepsilon_{3}^{\infty}\right)$ :

$$
w_{3}^{\prime}=\frac{1}{a}\left[\left(\omega_{1}^{0}\right)^{2} \varepsilon_{3}^{\infty}-\varepsilon_{3}^{0}\left(\omega_{3}^{0}\right)^{2}\right] \neq 0,
$$

\section{d. h. die Schwingung ist longitudinal.}

Zusammenfassend können wir sagen: Die Schwingungen in den beiden ausgezeichneten Richtungen, nämlich in der Richtung der Drehachse und senkrecht dazu, sind immer rein transversal oder longitudinal; es gelten die entsprechenden Formeln wie bei den Schwingungen in kubischen Kristallen, d. h. bei den transversalen Schwingungen sind die Frequenzen durch

$$
\omega_{1,2}=\omega_{\alpha}^{0},
$$

bei den longitudinalen durch

$$
\omega_{3}=\sqrt{\varepsilon_{\alpha}^{0} / \varepsilon_{\alpha}^{\infty} \omega_{\alpha}^{0}}
$$

gegeben, mit dem einen Unterschied, da 3 man noch durch einen Index $\alpha=1$ bzw. $\alpha=3$ zu unterscheiden hat, ob der Polarisationsvektor in der Ebene senkrecht zur Drehachse $\left(x_{1}, x_{2}\right.$-Ebene) oder in Richtung der Drehachse ( $x_{3}$-Richtung) liegt.

\section{Anhang}

\section{Herleitung der Grundgleichungen (4a,b), S. 48, aus der Energiedichte}

Bei den langen optischen Schwingungen bewegen sich die einzelnen Teilgitter starr gegeneinander, d. h. alle Atome desselben Teilgitters $k$ erleiden dieselbe Verrückung $\mathfrak{u}(k)$. Wenn es sich um geladene Atome handelt, so wird dabei im allgemeinen ein makroskopisches elektrisches Feld $\mathfrak{E}$ im Innern des Kristalls erzeugt, die Energiedichte $u$ wird somit eine Funktion der Verrückungen $\mathfrak{u}(k)$ und des elektrischen Feldes $\mathfrak{E}, \mathrm{d}$. h. es ergibt sich, falls man höhere als quadratische Terme vernachlässigt ${ }^{7}$ :

\footnotetext{
7 Vgl. hierzu Anm. ${ }^{6}$, S. 265/266.
} 


$$
\begin{aligned}
u=\frac{1}{2} \sum_{k, k^{\prime}} \mathrm{u}(k) \cdot F\left(k k^{\prime}\right) \cdot \mathrm{u}\left(k^{\prime}\right) & (\mathrm{A}, 1) \\
& +\sum_{k} \mathrm{u}(k) \cdot F(k) \cdot \mathbb{E}-\frac{1}{2} \mathfrak{E} \cdot F \cdot \mathfrak{E} .
\end{aligned}
$$

Das lineare Glied verschwindet, weil um die Gleichgewichtslage entwickelt wurde.

Für das Folgende ist wichtig, daß zwischen $F\left(k k^{\prime}\right)$ und $F(k)$ Beziehungen bestehen. Zunächst dürfen wir voraussetzen, daß die Koeffizienten in den rein quadratischen Gliedern von $(\mathrm{A}, \mathrm{l})$ (erster und dritter Ausdruck) symmetrisch sind, d. h.

$$
F\left(k^{\prime} k\right)=\overline{F\left(k k^{\prime}\right)}, \quad F=\bar{F} .
$$

(Der Querstrich bezeichnet die konjugierte Dyade.) Weitere Beziehungen ergeben sich aus der Forderung, daß sich die Energiedichte bei einer Bewegung des Kristalls als Ganzes (d. h. bei Festhalten der relativen Lage der Atome untereinander) nicht ändert. Ist die Bewegung eine kleine Translation, so ergibt sich:

$$
\sum_{k} F\left(k k^{\prime}\right)=\sum_{k^{\prime}} F\left(k k^{\prime}\right)=0, \sum_{k} F(k)=0 .
$$

(a)

$$
\text { (b) }
$$

Ferner wollen wir Deckoperationen

$$
\mathrm{r}(K)=\mathfrak{a}+T \cdot \mathrm{r}(k)
$$

$[\mathrm{r}(K)$ sind die Ortsvektoren des Teilgitters $K, \mathfrak{r}(k)$ die Ortsvektoren des Teilgitters $k]$

betrachten, bei denen das Gitter in sich selbst überführt wird. Dabei ist a ein Translationsvektor, $T$ beschreibt eine Drehung, Spiegelung oder eine Drehspiegelung. Dann ergibt sich:

$$
\begin{aligned}
F\left(K K^{\prime}\right) & =T \cdot F\left(k k^{\prime}\right) \cdot \bar{T}, \\
F(K) & =T \cdot F(k) \cdot \bar{T}, \\
F & =T \cdot F \cdot \bar{T} .
\end{aligned}
$$

Der Fall $K \neq k, K^{\prime} \neq k^{\prime}$ ist natürlich nur dann möglich, wenn die Teilgitter $K$ und $k$ als auch $K^{\prime}$ und $k^{\prime}$ gleichartig sind, weil sie sich ja sonst nicht ineinander überführen lassen. Für den Fall $K=k, K^{\prime}=k^{\prime}$ liefert die Gl. (A,5) Beziehungen zwischen den Komponenten der Dyaden:

$$
\begin{gathered}
F\left(k k^{\prime}\right)=T \cdot F\left(k k^{\prime}\right) \cdot \bar{T}, \\
F(k)=T \cdot F(k) \cdot \bar{T} .
\end{gathered}
$$

Setzen wir speziell voraus, daß nur zwei Teilgitter existieren, so gilt wegen $(\mathrm{A}, 3)$ :

$$
\begin{aligned}
F(11) & =-F(12)=-F(21)=F(22), \\
F(1) & =-F(2) .
\end{aligned}
$$

Durch Ableitung der Energiedichte nach $u(k)$ bzw. $\mathfrak{E}$ ergibt sich die Kraft $\Re_{k}$ auf das Teilgitter $k$ bzw. die

8 Vgl. hierzu ebenfalls Anm. ${ }^{6}$, S. 265/266.

$9 m_{k}$ : Masse eines Atoms der Sorte $k, v_{a}$ : Volumen der Elementarzelle. makroskopische Polarisation $\mathfrak{P}$. Benutzen wir noch die Newtonsche Bewegungsgleichung, so folgt ${ }^{8}$ :

$$
\begin{aligned}
\underline{o}_{k} \ddot{\mathfrak{u}}(k)=\mathfrak{\Re}_{k} & =-\frac{\partial u}{\partial \mathfrak{u}(k)} \\
& =-\sum_{k^{\prime}} F\left(k k^{\prime}\right) \cdot \mathfrak{u}\left(k^{\prime}\right)-F(k) \cdot \mathfrak{E},
\end{aligned}
$$

und $\mathfrak{P}=-\frac{\partial u}{\partial \mathfrak{E}}=-\sum_{k} \bar{F}(k) \cdot \mathfrak{u}(k)+F \cdot \mathfrak{E}$.

Dabei ist ${ }^{9} \varrho_{k}=m_{k} / v_{a}$ die Teilchendichte im Teilgitter $k$.

Die folgenden Gln. lassen sich bequemer schreiben, wenn wir

$$
\mathfrak{w}(k) \equiv \sqrt{\varrho} u(k)
$$

als reduzierten Schwingungsvektor einführen. Dividieren wir (A, 7a) gleichzeitig durch $\sqrt{\varrho_{k}}$, so erhalten die Gln. (A, 7) die Form:

$$
\begin{aligned}
\ddot{\mathfrak{w}}(k) & =-\sum_{k^{\prime}} \frac{F\left(k k^{\prime}\right)}{\varrho k} \cdot \mathfrak{w}\left(k^{\prime}\right)-\frac{F(k)}{\sqrt{\varrho k}} \cdot \mathfrak{E}, \\
\mathfrak{P} & =-\sum_{k} \frac{\frac{F(k)}{\sqrt{\varrho k}}}{\sqrt{\varrho_{k}}} \cdot \mathfrak{w}(k)+F \cdot \mathfrak{E} .
\end{aligned}
$$

Wir betrachten zunächst den einfachsten Fall, daß nur zwei Teilgitter existieren [besteht das Gitter aus nur einem Teilgitter (primitives Gitter), so sind die Atome immer ungeladen, d. h. $\mathfrak{F}$ und $\mathfrak{P}=0$ ]. Obwohl dieser Fall zwar in der Natur wohl nicht verwirklicht ist, lassen sich die prinzipiellen Gesichtspunkte hier am besten durchschauen, und er soll daher auch als Einführung in den allgemeineren Fall dienen.

Wir subtrahieren in $(\mathrm{A}, 9 \mathrm{a})$ die $\mathrm{Gl}$. für das Teilgitter 2 von der für das Teilgitter 1 :

$$
\begin{array}{r}
\ddot{\mathfrak{w}}(1)-\ddot{\mathfrak{w}}(2)=-\left(\frac{F(11)}{\varrho_{1}}-\frac{F(21)}{\varrho_{2}}\right) \cdot \mathfrak{w}(1) \quad(\mathrm{A}, 10) \\
+\left(\frac{F(22)}{\varrho_{2}}-\frac{F(12)}{\varrho_{1}}\right) \cdot \mathfrak{w}(2)-\left(\frac{F(1)}{\sqrt{\varrho_{1}}}-\frac{F(2)}{\sqrt{\varrho_{2}}}\right) \cdot \mathfrak{E} .
\end{array}
$$

Wegen $(A, 6)$ dürfen wir $(A, 10)$ und $(A, 9 b)$ in der Form schreiben:

$$
\begin{aligned}
\ddot{\mathfrak{w}}(1)-\ddot{\mathfrak{w}}(2)=\left(\frac{1}{\varrho_{1}}\right. & \left.+\frac{1}{\varrho_{2}}\right) F(12) \cdot(\mathfrak{w}(1)-\mathfrak{w} \\
& +\left(\frac{1}{V \varrho_{1}}+\frac{1}{V \varrho_{2}}\right) F(2) \cdot \mathfrak{E},
\end{aligned}
$$

$\mathfrak{P}=\left(\frac{1}{\sqrt{\varrho_{1}}}+\frac{1}{\sqrt{\varrho_{2}}}\right) \overline{F(2)} \cdot(\mathfrak{w}(1)-\mathfrak{w}(2))+F \cdot \mathfrak{E}$

Kürzen wir nun $\mathrm{ab}$

$$
\begin{aligned}
\mathfrak{w} & \equiv \mathfrak{w}(1)-\mathfrak{w}(2), \quad B^{11} \equiv\left(\frac{1}{\varrho_{1}}+\frac{1}{\varrho_{2}}\right) F(12), \\
B^{12} & \equiv\left(\frac{1}{\sqrt{\varrho_{1}}}+\frac{1}{\sqrt{\varrho_{2}}}\right) F(2), \\
B^{21} & \equiv\left(\frac{1}{\sqrt{\varrho_{1}}}+\frac{1}{\sqrt{\varrho_{2}}}\right) \overline{F(2)}, \quad B^{22} \equiv F
\end{aligned}
$$


so erhalten die Gln. die Gestalt:

$$
\begin{aligned}
& \ddot{\mathfrak{w}}=B^{11} \cdot \mathfrak{w}+B^{12} \cdot \mathfrak{E}, \\
& \mathfrak{P}=B^{21} \cdot \mathfrak{w}+B^{22} \cdot \mathfrak{E} \text { mit } B^{21}=\overline{B^{12}} .
\end{aligned}
$$

Damit sind die Gln. (4 a, b) hergeleitet. Über die Gestalt der $B^{i k}$ läßt sich dabei folgende Aussage machen: Da nach Voraussetzung die $x_{3}$-Achse 3-zählig, 4-zählig oder 6-zählig, also

$$
\begin{gathered}
T_{120^{\circ}=}\left(\begin{array}{ccc}
-\frac{1}{2} & \frac{1}{2} \sqrt{3} & 0 \\
-\frac{1}{2} \sqrt{3} & -\frac{1}{2} & 0 \\
0 & 0 & 1
\end{array}\right), \quad T_{90^{\circ}}=\left(\begin{array}{rrr}
0 & 1 & 0 \\
-1 & 0 & 0 \\
0 & 0 & 1
\end{array}\right) \\
\text { bzw. } T_{60^{\circ}}=\left(\begin{array}{ccc}
\frac{1}{2} & \frac{1}{2} \sqrt{3} & 0 \\
-\frac{1}{2} \sqrt{3} & \frac{1}{2} & 0 \\
0 & 0 & 1
\end{array}\right)
\end{gathered}
$$

eine Deckoperation ist, folgt durch Einsetzen von $T$ in $\left(\mathrm{A}, 5^{\prime}\right)$ und Beachten der Definitions-Gln. (A, 12) für die $B^{i k}$ :

$$
B^{i k}=\left(\begin{array}{ccc}
B_{11}^{i k} & B_{12}^{i k} & 0 \\
-B_{12}^{i k} & B_{11}^{i k} & 0 \\
0 & 0 & B_{33}^{i k}
\end{array}\right)
$$

Da aber nach (A, 2) und (A, 6) alle $F\left(k k^{\prime}\right) \quad\left(k, k^{\prime}=1,2\right)$ und $F$ symmetrisch sind, vereinfacht sich $(\mathrm{A}, 15)$ für $B^{11}$ und $B^{22} \mathrm{zu}$ :

$$
B^{i k}=\left(\begin{array}{ccc}
B_{11}^{i k} & 0 & 0 \\
0 & B_{11}^{i k} & 0 \\
0 & 0 & B_{33}^{i k}
\end{array}\right)
$$

Wollen wir auch für $B^{12}$ und $B^{21}$ diese Gestalt verlangen, so müssen wir eine weitere Symmetrieforderung stellen, z. B. daß die $x_{2}, x_{3}$-Ebene (bzw. allgemeiner eine Ebene, welche die $x_{3}$-Achse entält) eine Spiegelebene ist.

Durch Einsetzen der zugehörigen Deckoperation

$$
T_{\mathrm{Sp} .}=\left(\begin{array}{ccc}
-1 & & \\
& 1 & \\
& & 1
\end{array}\right)
$$

in $\left(\mathrm{A}, 5^{\prime}\right)$ folgt dann nämlich, daß $B_{12}^{i k}=B_{21}^{i k}=0$. Damit haben alle $B^{i k}$ die in Gl. $(4 \mathrm{a}, \mathrm{b})$ vorausgesetzte Gestalt.

Wir betrachten nun den Fall, daß das Gitter zwar aus mehr als zwei Teilgittern besteht, daß diese Teilgitter sich aber in zwei Gruppen zusammenfassen lassen, von denen jede gleichviele mit gleichen Atomen besetzte Teilgitter enthält. Die Teilgitter jeder Gruppe sollen durch Deckoperationen ineinander überführbar sein. Die $F\left(k k^{\prime}\right)$ bzw. $F(k)$ transformieren sich dann nach $(\mathrm{A}, 5)$. Wir nehmen nun weiter an, daß die $F\left(k k^{\prime}\right)$ bzw. $F(k)$ bereits eine solche Gestalt besitzen, daß sich diese bei der Transformation (A, 5) nicht mehr ändert. Dann gilt, wenn wir die Teilgitter der ersten Gruppe in passender Reihenfolge mit $1,3,5, \ldots, 2 m-1$, die der zweiten mit $2,4,6, \ldots, 2 m$ durchnumerieren ${ }^{10}$;

$F\left(k k^{\prime}\right)=F\left(k \pm 2, k^{\prime} \pm 2\right)=F\left(k \pm 4, k^{\prime} \pm 4\right)=\ldots$,

$$
F(k)=F(k \pm 2)=F(k \pm 4)=\ldots,
$$

[Die Gln. (A, 17) sind dabei nach rechts soweit fortzusetzen, bis in $F\left(K K^{\prime}\right)$ und $F(K) \quad K$ bzw. $K^{\prime}$ die Zahl 1 unter- oder $2 m$ überschreitet.]

Wir summieren nun die Gln. (A, 9a) über die Teilgitter jeder Gruppe, wobei wir die Summe über $k^{\prime}$ aufspalten:

$$
\begin{aligned}
& \sum_{k=1,3 \ldots}^{2 m-1} \ddot{\mathfrak{w}}(k)=-\sum_{k^{\prime}=1,3 \ldots}^{2 m-1}\left(\sum_{k=1,3 \ldots}^{2 m-1} \frac{F\left(k k^{\prime}\right)}{\varrho k}\right) \cdot \mathfrak{w}\left(k^{\prime}\right)-\sum_{k^{\prime}=2,4 \ldots}^{2 m}\left(\sum_{k=1,3 \ldots}^{2 m-1} \frac{F\left(k k^{\prime}\right)}{\varrho k}\right) \cdot \mathfrak{w}\left(k^{\prime}\right)-\left(\sum_{k=1,3 \ldots}^{2 m-1} \frac{F(k)}{\sqrt{\varrho k}}\right) \cdot(\mathfrak{E}, \\
& \sum_{k=2,4 \ldots}^{2 m} \ddot{\mathfrak{w}}(k)=-\sum_{k^{\prime}=1,3 \ldots}^{2 m-1}\left(\sum_{k=2,4 \ldots}^{2 m} \frac{F\left(k k^{\prime}\right)}{\varrho k}\right) \cdot \mathfrak{w}\left(k^{\prime}\right)-\sum_{k^{\prime}=2,4}^{2 m}\left(\sum_{k=2,4 \ldots}^{2 m} \frac{F\left(k k^{\prime}\right)}{\varrho k}\right) \cdot \mathfrak{w}\left(k^{\prime}\right)-\left(\sum_{k=2,4 \ldots}^{2 m} \frac{F(k)}{\sqrt{\varrho k}}\right) \cdot(\mathfrak{E} .
\end{aligned}
$$

Ebenso spalten wir die Summe in $(\mathrm{A}, 9 \mathrm{~b})$ auf :

$$
\mathfrak{P}=-\sum_{k=1,3 \ldots}^{2 m-1} \frac{\overline{F(k)}}{\sqrt{\varrho_{k}}} \cdot \mathfrak{w}(k)-\sum_{k=2,4 \ldots}^{2 m} \frac{\overline{F(k)}}{\sqrt{\varrho k}} \cdot \mathfrak{w}(k)+F \cdot \mathfrak{E} .
$$

Wegen

$$
\varrho_{1}=\varrho_{3}=\ldots=\varrho_{2 m-1}, \quad \varrho_{2}=\varrho_{4}=\ldots=\varrho_{2 m}
$$

und $(\mathrm{A}, 17)$ gilt nun

für $k^{\prime}=1,3, \ldots, 2 m-1: \quad \sum_{k=1,3 \ldots}^{2 m-1} \frac{F\left(k k^{\prime}\right)}{\varrho_{k}}=\frac{1}{\varrho_{1}} \sum_{k=1,3 \ldots}^{2 m-1} F(k 1), \quad \sum_{k=2,4 \ldots}^{2 m} \frac{F\left(k k^{\prime}\right)}{\varrho_{k}}=\frac{1}{\varrho_{2}} \sum_{k=2,4}^{2 m} F(k 1)$,

für $k^{\prime}=2,4, \ldots, 2 m$ :

$$
\sum_{k=1,3 \ldots}^{2 m-1} \frac{F\left(k k^{\prime}\right)}{\varrho_{k}}=\frac{1}{\varrho_{1}} \sum_{k=1,3 \ldots}^{2 m-1} F(k 2), \quad \sum_{k=2,4 \ldots}^{2 m} \frac{F\left(k k^{\prime}\right)}{\varrho_{k}}=\frac{1}{\varrho_{2}} \sum_{k=2,4 \ldots}^{2 m} F(k 2),
$$


d. h. die Summen sind von den $k^{\prime}$ aus ein und derselben Gruppe unabhängig. Ebenso folgt

$$
\sum_{k=1,3 \ldots}^{2 m-1} \frac{F(k)}{\sqrt{\varrho_{k}}}=\frac{m}{\sqrt{\varrho_{1}}} F(1), \quad \sum_{k=2,4}^{2 m} \frac{F(k)}{\sqrt{\varrho_{k}}}=\frac{m}{\sqrt{\varrho_{2}}} F(2) .
$$

Wenn wir jetzt $(A, 18 b)$ von $(A, 18 a)$ subtrahieren, so dürfen wir also schreiben:

$$
\begin{aligned}
\sum_{k=1,3 \ldots}^{2 m-1} \ddot{\mathfrak{w}}(k)-\sum_{k=2,4 \ldots}^{2 m} \ddot{\mathfrak{w}}(k)=\left(\frac{1}{\varrho_{2}} \sum_{k=2,4 \ldots}^{2 m} F(k 1)-\frac{1}{\varrho_{1}} \sum_{k=1,3 \ldots}^{2 m-1} F(k 1)\right) \cdot\left(\sum_{k^{\prime}=1,3 \ldots}^{2 m-1} \mathfrak{w}\left(k^{\prime}\right)\right) \\
+\left(\frac{1}{\varrho_{\mathbf{2}}} \sum_{k=2,4 \ldots}^{2 m} F(k 2)-\frac{1}{\varrho_{1}} \sum_{k=1,3 \ldots}^{2 m-1} F(k 2)\right) \cdot\left(\sum_{k^{\prime}=2,4 \ldots}^{2 m} \mathfrak{w}\left(k^{\prime}\right)\right)+m\left(\frac{F(2)}{\sqrt{\varrho_{2}}}-\frac{F(1)}{\sqrt{\varrho_{1}}}\right) \cdot \mathfrak{E} .
\end{aligned}
$$

Zur weiteren Umformung der Gln. bemerken wir, daß nach (A, 3a) gilt:

$$
\sum_{k=1,3 \ldots}^{2 m-1} F(k 1)=-\sum_{k=2,4 \ldots}^{2 m} F(k 1), \quad \sum_{k=1,3 \ldots}^{2 m-1} F(k 2)=-\sum_{k=2,4 \ldots}^{2 m} F(k 2) .
$$

Außerdem benutzen wir Gl. (A, 3b) : $\quad \sum_{k^{\prime}=1,3 \ldots}^{2 m-1} F\left(k k^{\prime}\right)=-\sum_{k^{\prime}=2,4 \ldots}^{2 m} F\left(k k^{\prime}\right)$,

woraus wir folgern können:

$$
\begin{aligned}
\sum_{k^{\prime}=1,3 \ldots}^{2 m-1}\left(\sum_{k=1,3 \ldots}^{2 m-1} F\left(k k^{\prime}\right)\right)= & -\sum_{k^{\prime}=2,4 \ldots}^{2 m}\left(\sum_{k=1,3 \ldots}^{2 m-1} F\left(k k^{\prime}\right)\right), \quad \sum_{k=1,3 \ldots}^{2 m-1} F(k 1)=-m \sum_{k=1,3 \ldots}^{2 m-1} F(k 2), \\
& \sum_{k=1,3 \ldots}^{2 m-1} F(k 1)=-\sum_{k=1,3 \ldots}^{2 m-1} F(k 2) .
\end{aligned}
$$

Im ersten Schritt wurde dabei wieder (A, 17 a) benutzt. Aus (A, 3 c) und (A, 17 b) folgt außerdem:

$$
F(1)=-F(2) \text {. }
$$

Unter Benutzung von $(\mathrm{A}, 23)$, (A, 24) und $(\mathrm{A}, 25)$ können wir $(\mathrm{A}, 22)$ und $(\mathrm{A}, 19)$ jetzt in der Form schreiben:

$$
\begin{gathered}
\sum_{k=1,3 \ldots}^{2 m-1} \ddot{\mathfrak{w}}(k)-\sum_{k=2,4 \ldots}^{2 m} \ddot{\mathfrak{w}}(k)=\left(\frac{1}{\varrho_{1}}+\frac{1}{\varrho_{2}}\right) \sum_{k=1,3}^{2 m} F(k 2) \cdot\left(\sum_{k^{\prime}=1,3 \ldots}^{2 m-1} \mathfrak{w}\left(k^{\prime}\right)-\sum_{k^{\prime}=2,4}^{2 m} \mathfrak{w}\left(k^{\prime}\right)\right)+m\left(\frac{1}{\sqrt{\varrho_{1}}}+\frac{1}{\sqrt{\varrho_{2}}}\right) F(2) \cdot \mathfrak{E} . \\
\mathfrak{P}=m\left(\frac{1}{\sqrt{\varrho_{1}}}+\frac{1}{\sqrt{\varrho_{2}}}\right) \overline{F(2)} \cdot\left(\sum_{k=1,3 \ldots}^{2 m-1} \mathfrak{w}(k)-\sum_{k=2,4 \ldots}^{2 m} \mathfrak{w}(k)\right)+F \cdot \mathfrak{E}
\end{gathered}
$$

Führen wir schließlich die Abkürzungen ein :

$$
\begin{gathered}
\mathfrak{w} \equiv \sum_{k=1,3 \ldots}^{2 m-1} \mathfrak{w}(k)-\sum_{k=2,4 \ldots}^{2 m} \mathfrak{w}(k), \quad B^{11} \equiv\left(\frac{1}{\varrho_{1}}+\frac{1}{\varrho_{2}}\right) \sum_{k=1,3 \ldots}^{2 m-1} F(k 2), \quad B^{12} \equiv m\left(\frac{1}{\sqrt{\varrho_{1}}}+\frac{1}{\sqrt{\varrho_{2}}}\right) F(2), \\
B^{21} \equiv m\left(\frac{1}{\sqrt{\varrho_{1}}}+\frac{1}{\sqrt{\varrho_{2}}}\right) F(2), \quad B^{22}=F,
\end{gathered}
$$

so erhalten die Gln. (A, 26) die Gestalt von $(4 \mathrm{a}, \mathrm{b})$ :

$$
\begin{aligned}
& \ddot{\mathfrak{w}}=B^{11} \cdot \mathfrak{w}+B^{12} \cdot \mathfrak{E}, \\
& \mathfrak{P}=B^{21} \cdot \mathfrak{w}+B^{22} \cdot \mathfrak{E} \quad \text { mit } \quad B^{21}=\overline{B^{12}} .
\end{aligned}
$$

Damit die $B^{i k}$ die in $(4, \mathrm{a}, \mathrm{b})$ geforderte Diagonalgestalt besitzen, genügt es, diese Gestalt für $F\left(k k^{\prime}\right)$, $F(k)$ und $F$ vorauszusetzen. Dann gilt auch wieder $B^{21}=B^{12}$.

$10 n=2 m$ ist dabei die Zahl der Teilgitter.
Schließlich sei als Beispiel eines Gitters, für das die obigen Voraussetzungen erfüllt sind, das Wurtzitgitter angeführt. Um die Gültigkeit der Voraussetzungen zu zeigen, sei kurz etwas über den Aufbau des Gitters gesagt. Das Wurtzitgitter kann man sich dadurch aufgebaut denken, daß man zwei mit verschiedenen Atomen besetzte Gitter hexagonal dichtester Kugelpackung (siehe Abb. $1 \mathrm{a}$ und $1 \mathrm{~b}$ ) gegeneinander verschiebt, und zwar etwa das zweite gegenüber dem ersten um $\frac{5}{5} a_{3}$. Die drei Basisvektoren $a_{1}, a_{2}, a_{3}$ spannen dabei die Elementarzelle auf. Da das Gitter hexagonal dichtester Kugel- 


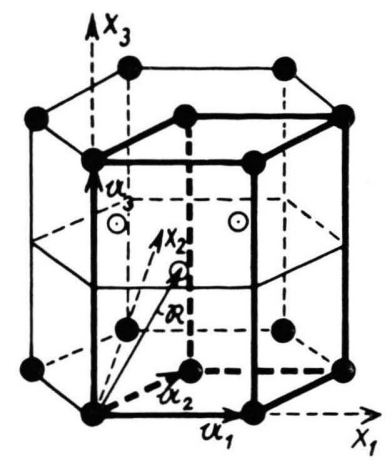

Abb. 1 a. Lage der Elementarzelle im Gitter hexagonal dichtester Kugelpackung (Elementarzelle breit ausgezogen).

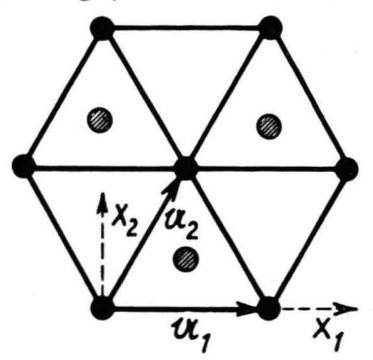

Abb. 1 b. Relative Lage der Atome zweier benachbarter Schichten im Gitter hexagonal dichtester Kugelpackung.

packung bereits aus zwei, und zwar mit gleichen Atomen besetzten Teilgittern besteht, die Elementarzelle also zwei Atome enthält, besteht das Wurtzitgitter aus vier Teilgittern, seine Elementarzelle enthält somit vier Atome (vgl. Abb. 2). Bezeichnet man die zum ersten Gitter hexagonal dichtester Kugelpackung gehörigen Teilgitter mit 1 und 3 (besetzt z. B. mit Zn-Atomen), die zum zweiten gehörigen mit 2 und 4 (besetzt z. B. mit S-Atomen), so sieht man leicht, daß die geforderten Voraussetzungen erfüllt sind:

Die Teilgitter 1 und 3 bzw. 2 und 4 lassen sich durch die Translation

$$
a=-\frac{1}{3}\left(a_{1}+a_{2}\right)-\frac{1}{2} a_{3}
$$

mit anschließender Spiegelung an der $x_{3}$-Achse

$$
T_{x_{3}}=\left(\begin{array}{lll}
-1 & & \\
& -1 & \\
& & 1
\end{array}\right)
$$

ineinander überführen.

Nun ist jede Achse in $x_{3}$-Richtung durch einen Gitterpunkt eine dreizählige Drehachse, wobei die Drehung

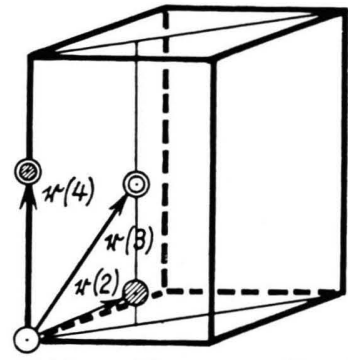

Atom aus dem:

Teilgitter 1$\}$

(C) Teilgitter 3$\}$

Z.B. Zn

Teilgitter 2 2 z.B.S

Abb. 2. Elementarzelle mit ihren 4 Gitterpunkten.

durch

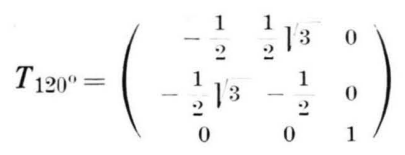

gegeben wird. Außerdem ist die $x_{2}, x_{3}$-Ebene eine Spiegelebene:

$$
T_{\mathrm{Sp} .}=\left(\begin{array}{lll}
-1 & & \\
& +1 & \\
& & +1
\end{array}\right)
$$

Aus der Existenz dieser beiden Symmetrieoperationen $T_{120^{\circ}}$ und $T_{\text {Sp. }}$ folgt bereits - wie man durch Einsetzen in (A, $5^{\prime}$ a) sieht (vgl. auch S. 56) -, daß die $F\left(k k^{\prime}\right)$ und $F(k)$ die Diagonalgestalt

$$
F=F_{1} \mathrm{i} \mathrm{i}+F_{1} \mathrm{i} \mathrm{i}+F_{3} \mathfrak{k}
$$

besitzen. Führen wir nun mit Hilfe der oben erwähnten Deckoperation $T_{x_{3}}$ die Teilgitter ineinander über, so ergibt sich nach (A, 5a) :

$$
F\left(k+2, k^{\prime}+2\right)=T_{x_{3}} \cdot F\left(k k^{\prime}\right) \cdot T_{x_{3}}=F\left(k k^{\prime}\right) .
$$

Letzteres gilt, da die Gestalt $(\mathrm{A}, 29)$ durch $T_{x_{3}}$ offenbar nicht mehr geändert wird. Die Voraussetzung (A, 17) ist also erfüllt, d. h. die Gln. (4) bzw. (A, 28) gelten auch für das Wurtzitgitter. Dabei ist [vgl. (A, 27)]:

$$
\begin{aligned}
\mathfrak{w} & =\mathfrak{w}(1)+\mathfrak{w}(3)-\mathfrak{w}(2)-\mathfrak{w}(4), \\
B^{11} & =\left(\frac{1}{\varrho_{1}}+\frac{1}{\varrho_{2}}\right)(F(12)+F(32)), \\
B^{12} & =B^{21}=\left(\frac{1}{\sqrt{\varrho_{1}}}+\frac{1}{V \varrho_{2}}\right) F(2), B_{22}=F .
\end{aligned}
$$

Herrn Prof. Dr. W. Franz möchte ich für die Förderung der Arbeit, für wertvolle Anregungen und Diskussionen herzlich danken. 